\title{
NOVO ADSORVENTE NANOESTRUTURADO HÍBRIDO DO TIPO AMINA/KIT-6 PARA CAPTURA DE CO
}

\author{
Fernando R. D. Fernandes ${ }^{\mathrm{a}, *, \odot}$, Francisco G. H. S. Pinto ${ }^{\mathrm{a}}$, Jhonny Villarroel-Rocha ${ }^{\mathrm{b}}$, Karim Sapag ${ }^{\mathrm{b}}$, Luiz D. Souza ${ }^{\mathrm{a}, \boldsymbol{}}$, \\ Vinícius P. S. Caldeira e Anne G. D. Santos ${ }^{a}$ \\ ${ }^{a}$ Departamento de Química, Universidade do Estado do Rio Grande do Norte, 59610-210 Mossoró - RN, Brasil \\ ${ }^{b}$ Laboratorio de Sólidos Porosos, Instituto de Física Aplicada, Universidad Nacional de San Luis, CP:5700 San Luis, Argentina
}

Recebido em 26/11/2020; aceito em 05/05/2021; publicado na web em 15/06/2021

\begin{abstract}
NEW HYBRID NANOSTRUCTURED ADSORBENT TYPE AMINE/KIT-6 FOR $\mathrm{CO}_{2}$ CAPTURE. The present work functionalized KIT-6 mesoporous material with aminopropyltrimethoxysilane (APTMS) and tested its efficiency in $\mathrm{CO}_{2}$ capture. The KIT-6 material was synthesized by hydrothermal method and functionalized via excess solvent in APTMS concentrations of 5 and $15 \%$ to obtain the hybrid adsorbents. All adsorbent materials were characterized by XRD, TGA/DTGA, DSC, $\mathrm{N}_{2}$ adsorption/desorption, SEM and applied in the $\mathrm{CO}_{2}$ capture, varying the temperature and pressure. The KIT-6 mesoporous structure at the hybrid adsorbents was kept. Variations in textural properties denoted that the organic molecules were incorporated in mesoporous structure. In the experiments, both chemisorption and physisorption were observed, reaching the best values at $25^{\circ} \mathrm{C}$, indicating that the temperature influences the adsorption process. The experiments showed adsorbed amounts of up to $0.8 \mathrm{mmol} / \mathrm{g}$ at atmospheric pressure, displaying the efficiency of the chemisorption provided by the $\mathrm{CO}_{2}$ interaction with the hybrid adsorbents. At high pressures, a predominance of physisorption was observed, demonstrating a better performance for the KIT-6 support, due to the superior textural properties. Finally, a well-ordered and functional hybrid adsorbent was successfully developed for the adsorption of carbon dioxide.
\end{abstract}

Keywords: mesoporous adsorbent; KIT-6; adsorption; carbon dioxide.

\section{INTRODUÇÃo}

A emissão de gases do efeito estufa (GEE) é considerada a principal causa de mudanças climáticas que se manifestam através do aquecimento global e outras calamidades naturais. O consumo de combustíveis fósseis é uma das, principais, causas da emissão de grande quantidade de GEE na atmosfera. ${ }^{1}$ Devido a isso, grandes esforços são dedicados a desenvolver materiais e técnicas para a redução de emissões de gases do efeito estufa, especialmente, para a captura e armazenamento de $\mathrm{CO}_{2}$. Com isto, uma opção interessante é a técnica de adsorção utilizando materiais híbridos formados por um suporte inorgânico poroso nanoestruturado com grupos ativos orgânicos ligados em sua estrutura por ligações químicas, ou seja materiais funcionalizados. ${ }^{2}$

O material mesoporoso do tipo KIT-6 já se mostrou promissor para várias áreas da pesquisa, sendo relatados vários trabalhos nas áreas de catálise e adsorção devido, principalmente, à sua elevada área específica, boa estabilidade térmica, largo diâmetro médio de poro, espessura de parede por volta de 4-6 nm, estrutura simétrica cúbica tridimensional Ia3d, grande volume de poro, e rede interpenetrante bicontínua de canais..$^{2-4} \mathrm{~A}$ técnica de adsorção pode ocorrer de duas formas: física e química. Para serem usados na adsorção, geralmente, os materiais porosos nanoestruturados são funcionalizados, modificando sua superfície com grupos funcionais orgânicos, como por exemplo, aminas, que apresentam grande afinidade das aminas com moléculas de $\mathrm{CO}_{2} .{ }^{5}$ As aminas são grupos bastante utilizados em adsorção, sendo relatados vários estudos na literatura. ${ }^{6-8}$ Dentre as aminas utilizadas para fornecer centros ativos na superfície do material KIT-6 encontra-se a 3-Aminopropiltrimetoxisilano (APTMS). Recentemente, vários trabalhos relataram a eficiência desses adsorventes na captura de moléculas de $\mathrm{CO}_{2} \cdot{ }^{9,10} \mathrm{O}$ uso do material KIT-6 para a adsorção de $\mathrm{CO}_{2}$ é um assunto inovador e pouco abordado na comunidade científica. Liu et $a l .{ }^{4}$ funcionalizaram o KIT-6 com tetrapropilamônio para estudar a adsorção de $\mathrm{CO}_{2}$ e, mais recentemente, Kishor e Ghoshal ${ }^{11}$ utilizaram aminopropiltrietóxisilano (APTES) para o mesmo fim.

Diante do exposto, o presente trabalho desenvolveu um material adsorvente nanoestruturado do tipo KIT-6 com grupos amina APTMS ancorados em sua superfície obtendo um material hibrido funcionalizado e o aplicou na adsorção de $\mathrm{CO}_{2}$.

\section{PARTE EXPERIMENTAL}

\section{Síntese do material KIT-6}

O material KIT-6 foi sintetizado em condições ácidas através do método hidrotérmico, conforme descrito na literatura. ${ }^{12} \mathrm{~A}$ composição estequiométrica molar para a respectiva síntese foi: 1,000 TEOS; 0,017 P123; 1,83 HCl; $195 \mathrm{H}_{2} \mathrm{O} ; 1,31$ Butanol. A síntese de $200 \mathrm{~g}$ de gel consistiu basicamente em dissolver 4,92 $\mathrm{g}(0,0007 \mathrm{~mol})$ do direcionador orgânico P123 (Sigma-Aldrich) em 177 mL (9,82 mols) de água destilada e 7,65 mL (0,10 mol) de $\mathrm{HCl}$ (Vetec, 37\%) sob agitação por 6 horas a uma temperatura de $35{ }^{\circ} \mathrm{C}$. Logo após, adicionaram-se 6,10 $\mathrm{mL}(0,066 \mathrm{~mol})$ de butanol (Vetec, 99,4\%) e o sistema foi mantido na mesma temperatura sob agitação constante por uma hora. Logo após adicionaram-se $11,40 \mathrm{~mL}(0,05 \mathrm{~mol}) \mathrm{de}$ Tetraetilortosilicato, TEOS (Sigma-Aldrich, 98\%), mantendo-se o sistema nas mesmas condições por 24 horas. Em seguida, o gel foi colocado na estufa para tratamento hidrotérmico em condições estáticas a uma temperatura de $100{ }^{\circ} \mathrm{C}$ por $24 \mathrm{~h}$. Em sequência o material foi lavado com uma solução de etanol (Synth, 95\%) e HCl à $2 \%$ e seco à temperatura ambiente. Posteriormente, o material foi calcinado a $550{ }^{\circ} \mathrm{C}$ por 6 horas, em uma taxa de aquecimento de $10^{\circ} \mathrm{C}$ por minuto para a remoção do direcionador orgânico. 


\section{Funcionalização do KIT-6}

O suporte KIT-6 foi funcionalizado após a síntese com 3-aminopropiltrimetóxisilano (APTMS) (Sigma-Aldrich) através do método de impregnação por excesso de solvente. O método consistiu em dissolver a fonte de amina em $50 \mathrm{~mL}$ (2,77 mols) de água por aproximadamente 15 minutos e depois, adicionar $1 \mathrm{~g}$ do suporte KIT-6, e manter a mistura em agitação à temperatura ambiente por 30 minutos. Posteriormente, o material incorporado com amina foi seco em estufa a $80{ }^{\circ} \mathrm{C}$ por 2 horas. Foram incorporados valores teóricos de $5 \%$ e $15 \%$ de amina. As amostras obtidas após a funcionalização foram nomeadas como K X\%, em que Ké o suporte inorgânico KIT-6 e X corresponde à porcentagem de APTMS incorporada.

\section{Caracterizações dos materiais}

A técnica de difração de raios-x (DRX) foi empregada utilizando o equipamento Rigaku modelo Mini Flex II com radiações de $\mathrm{CuK} \alpha$, em uma voltagem de $30 \mathrm{kV}$ e corrente de tubo de $15 \mathrm{~mA}$. A abertura da fenda foi de $0,15^{\circ}$ com passo de $0,005^{\circ}$ e tempo de $0,4 \mathrm{~s}$, sendo os dados coletados em baixo ângulo numa faixa de $2 \theta$ de 0,5 a $3^{\circ}$.

As curvas das análises termogravimétricas (TGA/DTGA) e da calorimetria exploratória diferencial (DSC) foram obtidas no equipamento STA 449F3- Júpiter. Utilizaram-se, aproximadamente, $5 \mathrm{mg}$ de material em um cadinho de alumina, sendo as amostras submetidas a aquecimento de 25 a $700{ }^{\circ} \mathrm{C}$, com uma razão de aquecimento de $10{ }^{\circ} \mathrm{C} \mathrm{min}^{-1}$, sob atmosfera dinâmica de nitrogênio com fluxo de $25 \mathrm{~mL} \mathrm{~min}^{-1}$.

As isotermas de adsorção/dessorção de nitrogênio foram obtidas em um equipamento Micromeritics ASAP 2020. As amostras foram previamente desgaseificadas a $70{ }^{\circ} \mathrm{C}$ por 3 horas e em seguida a $100{ }^{\circ} \mathrm{C}$ por 7 horas. Após a desgaseificação foram submetidas à adsorção/dessorção de nitrogênio a $77 \mathrm{~K}$. No cálculo de área específica $\left(S_{\mathrm{BET}}\right)$ foi aplicado o método B.E.T. (Brunauer, Emmett e Teller), levando em consideração os critérios de Rouqueroul. ${ }^{13}$ Para estimar o diâmetro modal dos poros utilizou-se o método V.B.S. (Villarroel-Barrera-Sapag) e o Volume total $\left(V_{\mathrm{TP}}\right)$ dos poros foi obtido usando a regra de Gurvich em um valor de $p / p^{0}$ igual a 0,98 .

A imagem de microscopia eletrônica de varredura (MEV) foi obtida utilizando-se um equipamento MIRA3 FERG da Shimadzu operando a 10,0 kV. Para a realização da análise, a amostra foi previamente imersa em acetona, posta em ultrassom por 15 minutos (para promover a separação das partículas), depositada sobre uma fita adesiva de carbono e submetida uma fina camada de ouro para garantir uma boa resolução das imagens. A média do tamanho das partíiculas foi determinada através do Image J.

\section{Teste de adsorção de $\mathrm{CO}_{2}$}

Os testes de adsorção foram empregados para avaliar a capacidade de captura de $\mathrm{CO}_{2}$ dos materiais obtidos em um procedimento semelhante ao descrito por Garcés et al. ${ }^{14}$ utilizando o equipamento ASAP 2050 da Micromeritics. Para a execução dos testes foi utilizado cerca de $0,2 \mathrm{~g}$ de material. Os experimentos ocorreram nas temperaturas de 25,35 e $50{ }^{\circ} \mathrm{C}$, todos sob pressão absoluta que variou de 6 a $7500 \mathrm{mmHg}$. A pureza do $\mathrm{CO}_{2}$ utilizado nos teste foi de $99,995 \%$.

Para a melhor interpretação dos dados experimentais obtidos, foram aplicados os modelos matemáticos de Langmuir (Eq. 1) e de Freundlich (Eq. 2). ${ }^{14}$ Ambos são modelos matemáticos que possibilitam melhor comparação entre os valores e interpretação dos dados.

$$
C=\frac{\mathrm{Q} \max \mathrm{K} p}{1+\mathrm{K} p}
$$

Para C temos a quantidade adsorvida ( $\mathrm{mmol} / \mathrm{g})$, Qmax é a capacidade de adsorção da monocamada ( $\mathrm{mmol} / \mathrm{g})$, K é a constante e $p$ é a pressão $(\mathrm{mmHg})$.

$$
\mathrm{C}=\mathrm{K} f p^{1 / n}
$$

Para o modelo de Freundlich temos que C é a quantidade adsorvida (mmol/g), Kfé uma constate de equilíbrio e $p$ é a pressão $(\mathrm{mmHg})$.

\section{RESULTADOS E DISCUSSÃO}

\section{DRX}

A Figura 1 apresenta os difratogramas a baixo ângulo do suporte KIT-6 e dos materiais híbridos.

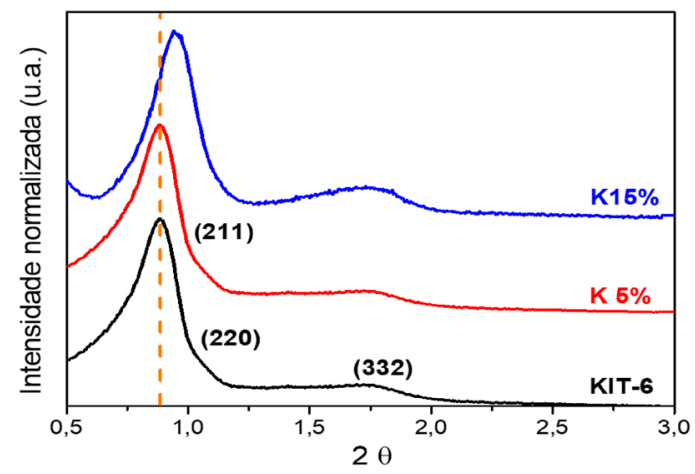

Figura 1. Difratogramas de raios X do suporte KIT-6 e dos materiais híbridos

Os difratogramas de raios- $\mathrm{X}$ apresentaram reflexões características de materiais do tipo KIT-6, com índices de Miller (211), (220) e (332). ${ }^{12,15,16}$ Esse resultado demonstra que mesmo após a funcionalização a estrutura do suporte foi mantida. Entretanto, nota-se que ao aumentar a quantidade de APTMS inserida ocorre um pequeno deslocamento no pico principal (211) na amostra K15\%, indicando uma interferência na mesoestrutura dos materiais e uma possível perturbação na estrutura do material em questão. Uma provável explicação para tal comportamento é o preenchimento da superfície e dos poros com compostos orgânicos, o que dificulta a difração através da estrutura mesoscópica. ${ }^{17}$

A Tabela 1 apresenta os valores das distâncias interplanares e do parâmetro mesoporoso do suporte KIT-6 e dos materiais híbridos. Pode-se observar uma diminuição no parâmetro mesoporoso quando se aumenta a quantidade de APTMS, sendo que a amostra K15\% apresentou o menor valor, indicando que a mesoestrutura sofreu alteração devido, provavelmente, a maior quantidade de amina inserida, corroborando com as discussões do DRX. Os resultados indicam que quanto maior a quantidade de amina, menor é o valor da distância interplanar e do parâmetro poroso dos materiais. Esse comportamento é um indicativo da presença da amina, tanto dentro dos poros, quanto na área externa dos materiais.

\section{TGA, DTGA e DSC}

A Figura 2 apresenta as curvas de TGA, DTGA e DSC de todas as amostras funcionalizadas.

Ao analisar as curvas TGA e DTGA, observa-se que o suporte apresentou apenas um evento de perda de massa quantificado 


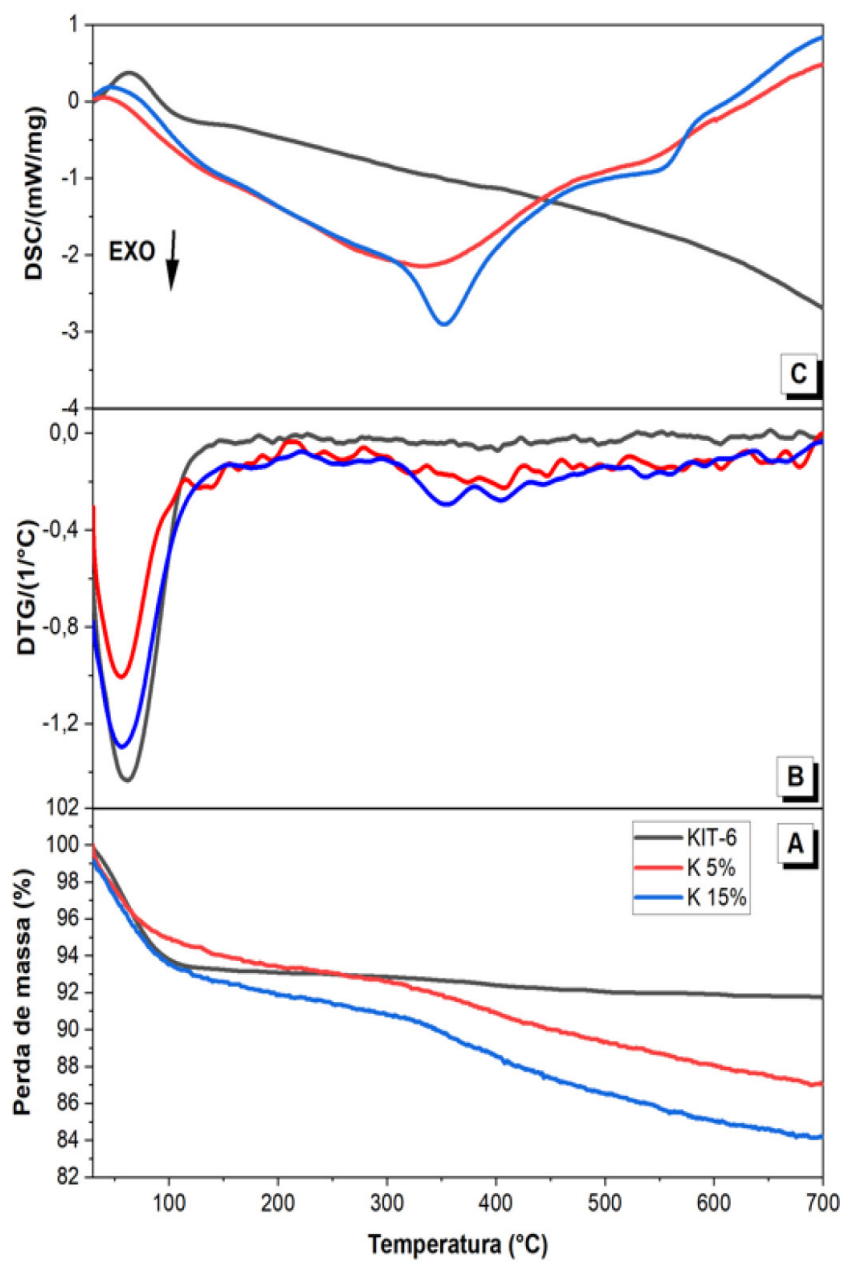

Figura 2. Curvas de: a) TGA; b) DTGA e c) DSC, de todos os materiais sitentizados

em 7,0\%, referente a saída de água fisissorvida em uma faixa de temperatura de $25^{\circ} \mathrm{C}$ à $110^{\circ} \mathrm{C}$. Os materiais adsorventes apresentaram dois eventos de perda de massa, no qual o primeiro evento, na faixa de $25^{\circ} \mathrm{C}$ à $110^{\circ} \mathrm{C}$, é referente à saída da água fisissorvida e o segundo evento, acima de $110^{\circ} \mathrm{C}$, é referente à degradação térmica e oxidativa da amina utilizada. Esses eventos térmicos se assemelham a trabalhos encontrados na literatura. ${ }^{18-20}$

As curvas de DSC corroboram com as análises de TGA e DTGA, demonstrando que o primeiro evento é endotérmico, referente a vaporização da água fisicamente adsorvida que absorve energia (para todos os materiais) e o segundo evento exotérmico, referente a degradação térmica e oxidativa da amina incorporada que libera energia.

Através dos dados obtidos na TGA também é possível determinar a porcentagem real de amina inserida, como relatado por Santos et al., ${ }^{21}$ em um estudo sobre impregnação de quitosana no
SBA-15. Os resultados obtidos na análise termogravimétrica indicam impregnações reais, significativas, sendo observadas quantidades de $7,9 \%$ e $9,1 \%$, para os materiais K5\%, e K15\%, respectivamente. Outra informação obtida foram os valores dos grupos aminos presentes nos materiais impregnados, com base nas porcentagens de amina obtidas pode-se determinar os valores de $0,81 \mathrm{mmol} \mathrm{N} / \mathrm{g} \mathrm{e} 1,32 \mathrm{mmol} \mathrm{N} / \mathrm{g}$, para as amostras $\mathrm{K} 5 \%$ e $\mathrm{K} 15 \%$, respectivamente. Esses resultados confirmam a presença dos grupos aminas no suporte inorgânico.

\section{Adsorção e dessorção de $\mathrm{N}_{2}$}

A Figura 3 apresenta as isotermas de adsorção/dessorção de $\mathrm{N}_{2}$ e as distribuições de diâmetro de poros de todos os materiais desenvolvidos. A Tabela 1 apresenta os valores determinados das propriedades texturais de todos os materiais obtidos.

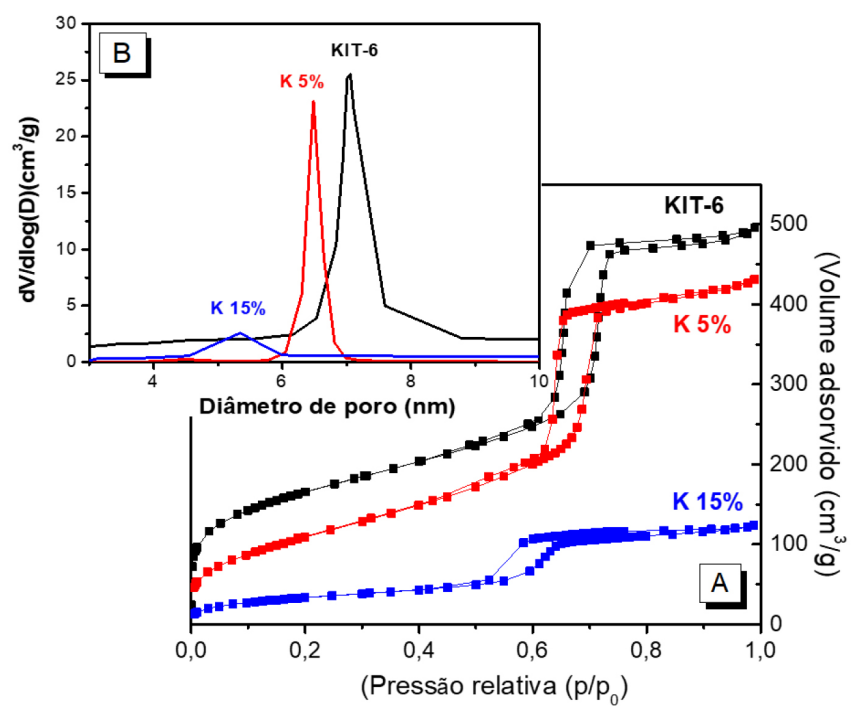

Figura 3. a) Isotermas de adsorção e dessorção de $\mathrm{N}_{2}$; b) distribuição de diâmetro de poros do suporte KIT-6 e dos materiais híbridos

Nos resultados apresentados na Figura 3a, observa-se que, de uma forma geral, todas as amostras apresentaram isotermas do Tipo IV (a), que pode ser atribuída a materiais mesoporosos, com loop de histerese do Tipo H1. Isso demonstra que os materiais formados possuem poros unidirecionais e bem organizados, corroborando com os resultados de DRX aqui apresentados. ${ }^{4,11,22}$ Nota-se um decréscimo no volume total adsorvido e uma modificação no perfil da isoterma, quando comparados os materiais com 5 e 15\%, o que está relacionado com a quantidade de amina inserida e ocorre devido à concentração de amina na superfície e nos poros dos materiais. Os resultados das isotermas reforçam os resultados já apresentados aqui de DRX e TGA em que foi possível observar uma leve desorganização do material a medida que se aumenta a quantidade de amina. Comportamento semelhante foi encontrado por Kishor e Ghoshal, ${ }^{11}$ ao funcionalizarem o KIT-6 com 3-aminopropiltrietóxisilano (APTES).

Tabela 1. Valores da distância interplanar, parâmetro mesoporoso e propriedades texturais do suporte KIT-6 e dos materiais híbridos

\begin{tabular}{cccccccc}
\hline Amostras & $\begin{array}{c}\mathrm{d}_{(211)} \\
(\mathrm{nm})\end{array}$ & $\begin{array}{c}\mathrm{a}_{0} \\
(\mathrm{~nm})\end{array}$ & $\begin{array}{c}S_{\text {BET }} \\
\left(\mathrm{m}^{2} / \mathrm{g}\right)\end{array}$ & $\begin{array}{c}V_{\text {TP }} \\
\left(\mathrm{cm}^{3} / \mathrm{g}\right)\end{array}$ & $\begin{array}{c}V_{\text {MESO }} \\
\left(\mathrm{cm}^{3} / \mathrm{g}\right)\end{array}$ & $\begin{array}{c}V_{\text {MICRO }} \\
\left(\mathrm{cm}^{3} / \mathrm{g}\right)\end{array}$ & $\begin{array}{c}D_{\mathrm{P}} \\
(\mathrm{nm})\end{array}$ \\
\hline KIT-6 & 5,14 & 12,60 & 603 & 0,76 & 0,68 & 0,02 & 7,0 \\
K5\% & 5,13 & 12,56 & 349 & 0,56 & 0,47 & 0,00 \\
K15\% & 4,70 & 11,51 & 124 & 0,19 & 0,11 & 0,00 & 6,5 \\
\hline
\end{tabular}

$\mathrm{d}_{211}=$ Distancia interplanar; $\mathrm{a}_{0}=$ Parâmetro da célula unitária para estrutura cúbica obtida através da equação $\mathrm{a}_{0 \text {-Cúbica }}=\mathrm{d}_{211} 6^{1 / 2} ; S_{\text {BET }}=$ Área específica (determinada através do cálculo de BET); $V_{\mathrm{TP}}=$ Volume de poros; $V_{\mathrm{MESO}}=$ Volume de mesoporos primários; $V_{\text {Micro }}=$ Volume microporoso; $D_{\mathrm{P}}=$ Diâmetro modal de poros (determinado através da distribuição de tamanho de poro obtida com o método VBS). 
Analisando os valores apresentados na Tabela 1, observa-se que o aumento na concentração de amina impregnada afetou de forma significativa as propriedades texturais dos materiais funcionalizados. Nota-se um comportamento decrescente nos valores da área específica, diâmetro de poros, volume total de poros e volume dos mesoporos primários. Essa correlação é um indicativo de que a amina impregnada está, não apenas na superfície, fora dos mesoporos primários, mas também dentro dos poros, visto que o volume de mesoporos diminui à medida que aumenta a \% de amina. Como consequência da funcionalização tem-se o preenchimento total ou bloqueio dos microporos, como pode ser visto nos resultados de

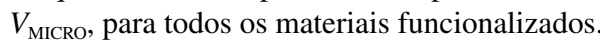

O aumento no valor do diâmetro modal de poro $\left(D_{\mathrm{P}}\right)$ da amostra K5\% pode ser explicado devido ao preenchimento parcial dos poros com amina, deslocando o perfil de distribuição para maiores valores do diâmetro de poros. Já para a amostra K15\% o diâmetro foi de $5,3 \mathrm{~nm}$, comportamento já esperado, pois uma maior quantidade de material foi inserido no suporte diminuindo seu tamanho e, consequentemente, também, a área e o volume de poros, resultados semelhantes podem ser vistos na literatura. ${ }^{11,23}$

\section{MEV}

A Figura 4 apresenta, de forma representativa, a imagem de MEV do suporte KIT-6.

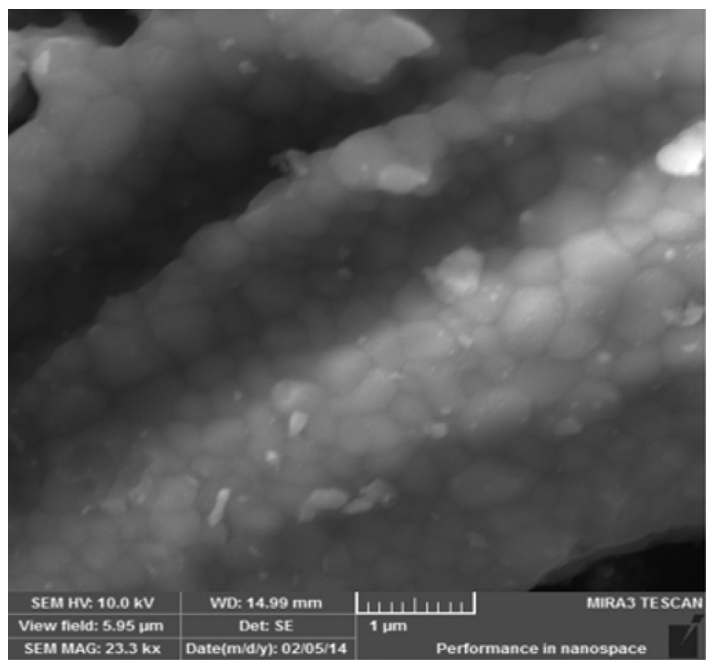

Figura 4. Imagem de MEV do suporte KIT-6

Ao analisar a imagem do suporte KIT-6 é possível notar que a amostra é composta por aglomerados de nanopartículas, apresentando formas e tamanhos irregulares. ${ }^{24,25}$ Através do tratamento com o Image $\mathbf{J}$ foi possível determinar de forma qualitativa o tamanho das partículas, observando-se valores em escala nanométrica, que variaram entre 100 e $700 \mathrm{~nm}$. Esse comportamento corrobora e complementa os dados de DRX e adsorção e desorção de $\mathrm{N}_{2}$, demonstrando de fato a síntese de um material nanoestruturado.

\section{Testes de adsorção de $\mathrm{CO}_{2}$}

As Figuras 5, 6 e 7 apresentam as isotermas de adsorção de $\mathrm{CO}_{2}$ a 25,35 e $50{ }^{\circ} \mathrm{C}$, para o suporte KIT-6 e para os materiais híbridos.

Ao observar as isotermas de adsorção de $\mathrm{CO}_{2}$ em diferentes temperaturas, nota-se que os testes realizados a $25^{\circ} \mathrm{C}$ apresentaram os melhores resultados a baixa e a altas pressões, apresentando adsorções de $0,80 \mathrm{mmol} / \mathrm{g}$ em $760 \mathrm{mmHg}$ até $3,3 \mathrm{mmol} / \mathrm{g} \mathrm{em}$ $7500 \mathrm{mmHg}$. Os resultados obtidos a $35^{\circ} \mathrm{C}$ e a $50^{\circ} \mathrm{C}$, apresentaram



Figura 5. a) Isotermas de adsorção de $\mathrm{CO}_{2}$ do suporte KIT-6 e dos materiais híbridos a $25^{\circ} \mathrm{C}$; b) em destaque a adsorção em baixas pressões

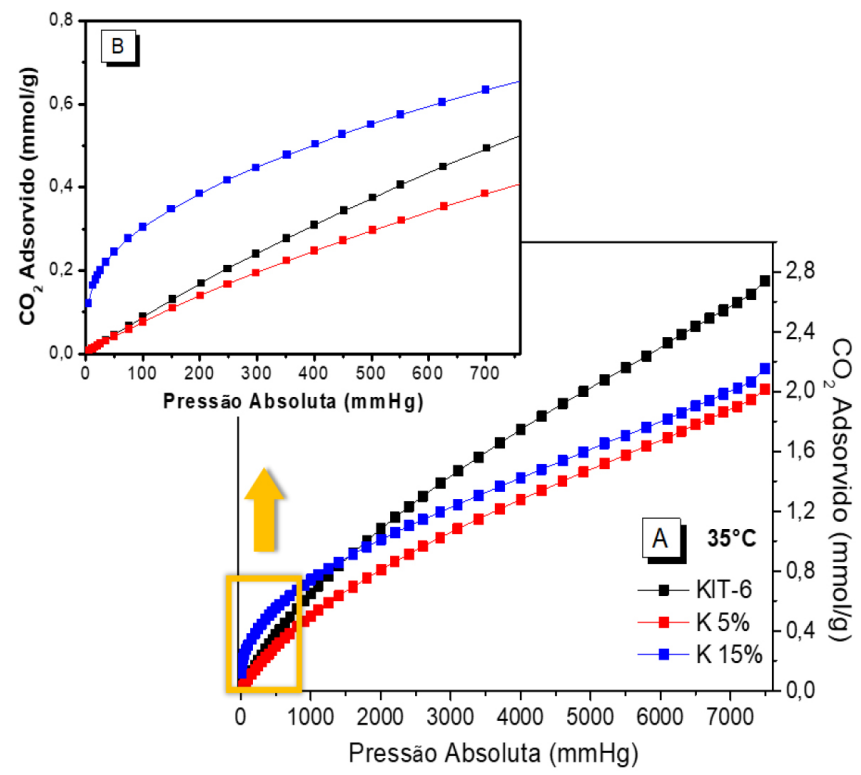

Figura 6. a) Isotermas de adsorção de $\mathrm{CO}_{2}$ do suporte KIT-6 e dos materiais híbridos a $35^{\circ} \mathrm{C}$; b) em destaque a adsorção em baixas pressões

comportamentos semelhantes, entretanto, obtiveram-se valores de adsorção consideravelmente inferiores. Esses resultados evidenciam que a temperatura de $25{ }^{\circ} \mathrm{C}$ é a melhor temperatura de adsorção de $\mathrm{CO}_{2}$ para os adsorventes utilizados. Resultados semelhantes foram encontrados por Heydari-Gorji e Sayari, ${ }^{26}$ ao trabalharem com Polietilenamina (PEI). Os autores afirmam que temperaturas mais baixas favorecem a adsorção de $\mathrm{CO}_{2}$ em materiais com baixa concentração de amina inserida (como é caso das concentrações utilizadas nos testes deste trabalho).

A baixa concentração de amina propicia uma melhor dispersão da mesma sobre o material, tornando os locais de adsorção mais facilmente acessíveis, pois, devido à natureza exotérmica da interação entre o $\mathrm{CO}_{2}$ e a amina não são necessárias temperaturas muito altas para promover tal reação. Corroborando com esse comportamento, os resultados observados a 35 e $50{ }^{\circ} \mathrm{C}$ mostram que a quantidade de $\mathrm{CO}_{2}$ adsorvida sofre um decréscimo significativo.

A Tabela 2 apesenta as quantidades de $\mathrm{CO}_{2}$ adsorvido nos 


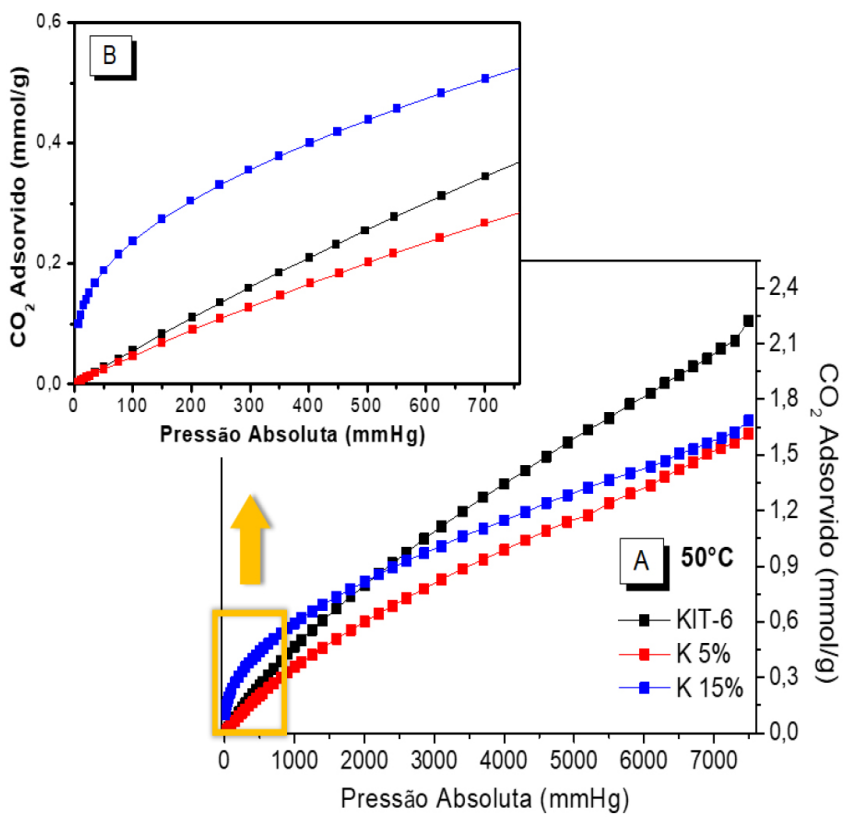

Figura 7. a) Isotermas de adsorção de $\mathrm{CO}_{2}$ do suporte KIT-6 e dos materiais híbridos $a 50^{\circ} \mathrm{C}$; b) em destaque a adsorção em baixas pressões

materiais híbridos a baixa e a altas pressões, em função da quantidade real de amina impregnada.

Os resultados de adsorção de $\mathrm{CO}_{2}$ obtidos a $25^{\circ} \mathrm{C}$ (melhor condição de adsorção) apresentaram as melhores capacidades de adsorção a $760 \mathrm{mmHg}$, na qual a amostra $\mathrm{K} 15 \%$ apresentou a maior capacidade de adsorção $(0,80 \mathrm{mmol} / \mathrm{g})$ e uma melhor eficiência $(0,60)$ em comparação a amostra K5\%. Essa eficiência leva em consideração a estequiometria de $2 \mathrm{~mol}$ de $\mathrm{N}$ para $1 \mathrm{~mol} \mathrm{de} \mathrm{CO}_{2}{ }^{7}$ A altas pressões ocorreu comportamento inverso, sendo o material KIT-6 a que apresentou valores mais significativos $(3,3 \mathrm{mmol} / \mathrm{g})$. Esse comportamento pode ser explicado pelo fato de pressões mais baixas favorecerem a adsorção química (quimissorção) realizada através dos materiais funcionalizados, enquanto pressões mais altas favorecem a adsorção física (fisissorção), tipo de adsorção que é mais acentuada no material puro, conforme descrito no estudo de Sanz et al. ${ }^{27}$ Resultados semelhantes foram obtidos por Aziz e colaboradores em 2012, ${ }^{28}$ em que eles determinaram que em baixas pressões o $\mathrm{CO}_{2}$ tende a

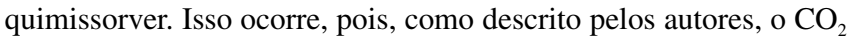
quimissorvido apresenta valores de entalpia de adsorção próximos a $98 \mathrm{~kJ} \mathrm{~mol}^{-1}$ apresentando maiores valores. Enquanto o $\mathrm{CO}_{2}$ fisissorvido apresenta valores de entalpia de adsorção próximos a $35 \mathrm{~kJ} \mathrm{~mol}^{-1} \mathrm{em}$ materiais de sílica mesoporosa.

Como visto nas propriedades texturais (Tabela 1), o suporte KIT6 apresentou maior área específica entre os adsorventes utilizados $\left(603 \mathrm{~m}^{2} / \mathrm{g}\right)$, o que propicia maior área para captura das moléculas de $\mathrm{CO}_{2}$, pincipalmente, a altas pressões. Para os materiais híbridos a área foi, consideravelmente, reduzida, mas devido à presença das aminas, as interações químicas entre o $\mathrm{CO}_{2}$ e os grupos amino (quimissorção) são mais evidenciadas, principalmente a baixas pressões, e sofrem um aumento, embora não tão significativo quanto no suporte KIT-6 a altas pressões.

Os resultados de adsorção de $\mathrm{CO}_{2}$ se mostraram bastante satisfatórios, pois se conseguiu uma boa capacidade de adsorção a baixa temperatura e pressão com um adsorvente com baixa concentração de amina impregnada. Outro resultado importante foi a capacidade de adsorção do material puro, mesmo a baixas pressões o material conseguiu adsorver quantidades semelhantes e até superiores a materiais do mesmo tipo relatados na literatura. ${ }^{2,11}$ Dessa forma, existem diferentes possibilidades, dependendo das condições de temperatura e pressão, que se deseja realizar a adsorção do $\mathrm{CO}_{2}$.

\section{Aplicação dos modelos de Freundlich e Langmuir}

A Tabela 3 apresenta os valores obtidos a partir dos modelos matemáticos de Freundlich e Langmuir, aplicados nas isotermas de adsorção de $\mathrm{CO}_{2}$, e a Figura 8 apresenta, de forma representativa, as isotermas da amostra $\mathrm{K} 15 \%$ à $25^{\circ} \mathrm{C}$ após os tratamentos matemáticos.

Avaliando os valores de $\mathrm{R}^{2}$ para os modelos de Freundlich e Langmuir é possível notar que o modelo de Freundlich apresentou os melhores ajustes aos valores experimentais, apresentando valores entre 0,9934 - 09996. Esses valores demonstram uma boa correlação do modelo aplicado com as isotermas experimentais. Por esse motivo

Tabela 2. Valores das quantidades de $\mathrm{CO}_{2}$ adsorvido em baixas e altas pressões na temperatura de $25^{\circ} \mathrm{C}$

\begin{tabular}{ccccc}
\hline Amostras & $\begin{array}{c}\text { Quantidade real de amina } \\
\text { impregnada }(\%)\end{array}$ & $\begin{array}{c}760 \mathrm{mmHg} \\
(\mathrm{mmol} / \mathrm{g})\end{array}$ & $\begin{array}{c}7500 \mathrm{mmHg} \\
(\mathrm{mmol} / \mathrm{g})\end{array}$ & $\begin{array}{c}\text { Eficiência } \\
\left(\mathrm{CO}_{2} / \mathrm{N}\right)^{\mathrm{a}}\end{array}$ \\
\hline KIT-6 & - & 0,68 & 3,3 & - \\
K 5\% & 7,9 & 0,50 & 2,3 & 0,62 \\
K 15\% & 9,1 & 0,80 & 2,3 & 0,60 \\
\hline
\end{tabular}

${ }^{\mathrm{a}}$ Calculado a partir da relação de $\mathrm{CO}_{2}$ adsorvido $\left(\mathrm{mmol} \mathrm{CO}_{2} / \mathrm{g}\right)$ a baixas pressões e grupos amina presentes nos materiais híbridos (mmol N/g).

Tabela 3. Dados obtidos a partir dos modelos matemáticos de Freundlich e Langmuir

\begin{tabular}{|c|c|c|c|c|c|c|c|c|c|}
\hline & \multicolumn{9}{|c|}{ ADSORVENTES } \\
\hline & \multicolumn{3}{|c|}{$25^{\circ} \mathrm{C}$} & \multicolumn{3}{|c|}{$35^{\circ} \mathrm{C}$} & \multicolumn{3}{|c|}{$50^{\circ} \mathrm{C}$} \\
\hline & KIT-6 & $5 \%$ & $15 \%$ & KIT-6 & $5 \%$ & $15 \%$ & KIT-6 & $5 \%$ & $15 \%$ \\
\hline \multicolumn{10}{|l|}{ FREUNDLICH $^{a}$} \\
\hline $\mathrm{Kf}(\mathrm{mmol} / \mathrm{g})(\mathrm{mmHg})$ & 0,0074 & 0,0062 & 0,0426 & 0,0047 & 0,0042 & 0,0254 & 0,0022 & 0,0019 & 0,0212 \\
\hline $\mathrm{n}$ & 1,47 & 1,52 & 2,26 & 1,40 & 1,45 & 2,04 & 1,28 & 1,33 & 2,07 \\
\hline $\mathrm{R}^{2}$ & 0,9994 & 0,9993 & 0,9946 & 0,9994 & 0,9995 & 0,9934 & 0,9996 & 0,9995 & 0,9962 \\
\hline \multicolumn{10}{|l|}{ LANGMUIR $^{\mathrm{b}}$} \\
\hline Qmax (mmol/g) & 6,06 & 3,93 & 2,64 & 5,47 & 3,75 & 2,59 & 5,43 & 3,73 & 2,02 \\
\hline $\mathrm{K}(1 / \mathrm{mmHg})$ & $1,461 \mathrm{E}-4$ & $1,624 \mathrm{E}-4$ & $5,035 \mathrm{E}-4$ & $1,229 \mathrm{E}-4$ & $1,376 \mathrm{E}-4$ & $3,725 \mathrm{E}-4$ & $8,506 \mathrm{E}-4$ & $9,422 \mathrm{E}-4$ & $3,957 \mathrm{E}-4$ \\
\hline $\mathrm{R}^{2}$ & 0,9968 & 0,9938 & 0,9439 & 0,9977 & 0,9961 & 0,9520 & 0,9984 & 0,9973 & 0,9589 \\
\hline
\end{tabular}


Tabela 4. Comparação com alguns adsorventes presentes na literatura

\begin{tabular}{ccccccc}
\hline Adsorvente & Amina & Pressão & Temperatura $\left({ }^{\circ} \mathrm{C}\right)$ & $\mathrm{CO}_{2}$ adsorvido $(\mathrm{mmol} / \mathrm{g})$ & Referência \\
\hline KIT-6 & APTES & 1 bar & 30 & 0,76 & $(11)$ & $(11)$ \\
KIT-6 & APTES & 1 bar & 30 & 0,83 & $(31)$ \\
MCM-48 & APTES & 1 bar & 25 & 0,80 & $(32)$ \\
MCM-41 & APTES & - & 25 & 0,83 & $(32)$ \\
KIT-6 & PEI & - & 25 & 0,80 & Presente estudo \\
KIT-6 & APTMS & $760 \mathrm{mmHg}$ & 25 & 0,80 & \\
\hline
\end{tabular}

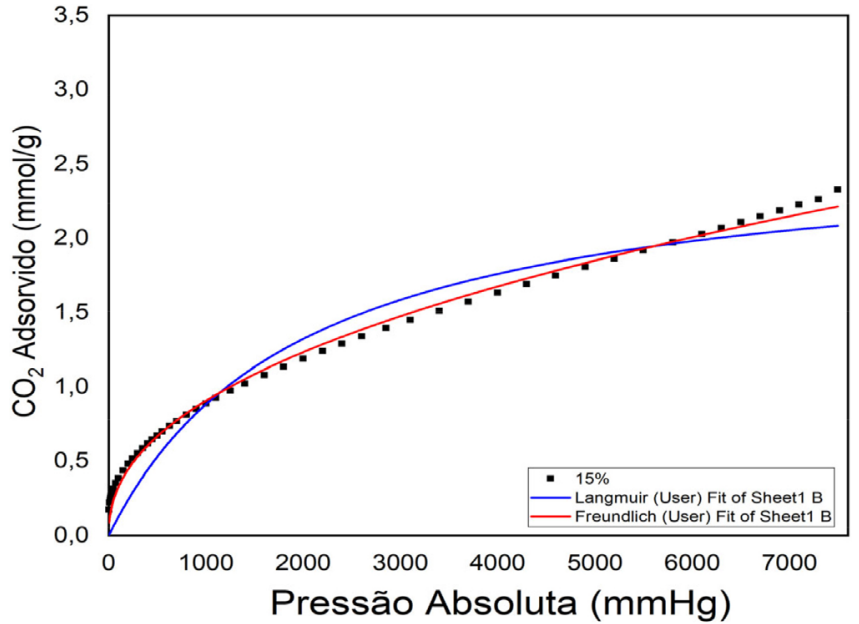

Figura 8. Isotermas de adsorção de $\mathrm{CO}_{2}$ nos modelos de Langmuir e Freundich da amostra $\mathrm{K} 15 \%$ a $25{ }^{\circ} \mathrm{C}$

será usado o modelo de Freundlich para discutir os dados obtidos. Para o modelo de Freundlich, os valores de $\mathrm{n}$ variam um pouco entre as amostras, porém, não excede o valor mínimo e máximo que é de 1 até $10 .{ }^{29}$ Segundo Delle-Site, $2001,{ }^{29}$ quando os valores de $\mathrm{n}$ variam entre 1 e 10 , eles representam condições favoráveis de adsorção, sendo que, quanto maior o valor de $n$, maior a interação entre o adsorvente e o adsorbato. Os valores de $\mathrm{n}$ para o suporte puro e os respectivos materiais com amina ( $5 \%$ e $15 \%$ ) estão como esperado, pois quanto maior a quantidade de amina, maiores serão as interações entre o adsorvente e o $\mathrm{CO}_{2}$. A amostra que apresentou maior valor de $\mathrm{n}$ foi a $\mathrm{K} 15 \%$ (2,26 para valor de $\mathrm{n})$, como pode ser visto na Tabela 3. Para todas as condições de testes de $\mathrm{CO}_{2}(25$, 35 e $50{ }^{\circ} \mathrm{C}$ ), o suporte contendo $15 \%$ de amina inserida foi o que apresentou os maiores valores $\mathrm{n}$. O Kf é um parâmetro que está relacionado diretamente com a capacidade de adsorção e o material contendo $15 \%$ de amina nas condições de $25^{\circ} \mathrm{C}$ apresentou o maior valor Kf. Comparando os valores dos experimentos em diferentes condições de temperatura, como, por exemplo, o suporte com $15 \%$ de amina, o valor de $\mathrm{Kf}$ vai decrescendo à medida que se aumenta $\mathrm{a}$ temperatura do experimento $\left(25>35>50^{\circ} \mathrm{C}\right)$. Tal comportamento está relacionado com a capacidade de adsorção do material diminuir à medida que a temperatura aumenta, devido ao processo de adsorção ser um processo exotérmico. ${ }^{30}$

Correlacionando os resultados obtidos, principalmente pelo material K15\% (1,16 mmol N/g), com alguns estudos semelhantes presentes na literatura, pode-se ter uma ideia do significativo desempenho obtido, como pode ser visto na Tabela 4.

Os resultados de adsorção de $\mathrm{CO}_{2}$ se mostraram bastante satisfatórios, pois se conseguiu uma boa capacidade de adsorção a baixa temperatura e pressão com um adsorvente com baixa concentração de amina impregnada. Dessa forma, existem diferentes possibilidades, dependendo das condições de temperatura e pressão, que se deseja realizar a adsorção do $\mathrm{CO}_{2}$.

\section{CONCLUSÕES}

Os resultados obtidos demonstram que a síntese do suporte KIT6 foi obtida com êxito e que a técnica de funcionalização utilizada resultou na inserção da amina sem destruir a estrutura cúbica do suporte KIT-6. As propriedades texturais comprovaram que a amina inserida se encontra tanto nos poros, quanto na superfície dos materiais estudados. Os testes de adsorção de $\mathrm{CO}_{2}$ foram significativos, tanto para os materiais funcionalizados, quanto para o suporte KIT-6. A adsorção numa temperatura constante $\left(25^{\circ} \mathrm{C}\right)$ depende do tipo de processo de sorção (física ou química), da área superficial, da \% de amina e da pressão de adsorção. A introdução de amina criou sitios de quimisorção, mas diminui a área para fisisorção, tornando esses processos competitivos entre si e dependentes da pressão. Em pressões baixas $(760 \mathrm{mmHg})$ prevaleceu a quimisorção e o \% adsorvido se deve, preferivelmente, à quantidade de amina inserida sendo maior no $\mathrm{K} 15 \%$, que tem a maior quantidade de amina. Em pressões altas prevaleceu a fisisorção e a \% adsorvida se deve, preferivelmente, à área superficial disponível, sendo maior para o suporte KIT-6, que tem a maior área superficial.

\section{AGRADECIMENTOS}

Os autores agradecem à Universidade do estado do Rio Grande do Norte, ao Laboratorio de Sólidos Porosos, à CAPES pela bolsa fornecida e ao professor L.D. Souza (in memorian) por todas as contribuições e ensinamentos.

\section{REFERÊNCIAS}

1. Lakhi, K. S.; Singh, G.; Kim, S.; Baskar, A. V.; Joseph, S.; Yang, J.-H.; Vinu, A.; Microporous Mesoporous Mater. 2018, 267, 134.

2. Zhang, Z.; Wang, H.; Chen, X.; Xie, R.; Gao, P.; Wei, W.; Sun, Y.; Adsorption 2014, 20, 883.

3. Dou, B.; Hu, Q.; Li, J.; Qiao, S.; Hao, Z.; J. Hazard. Mater. 2011, 186, 1615.

4. Liu, Y.; Liu, Y.; Shi, J.; Chen, J.; Ye, Q.; Pan, H.; Shao, Z.; Shi, Y.; Microporous Mesoporous Mater. 2010, 134, 16.

5. Chen, C.; Kim, J.; Ahn, W.; Korean J. Chem. Eng. 2014, 31, 1919.

6. Vieira, R. B.; Pastore, H. O.; Dalton Trans. 2018, 47, 3102.

7. Vieira, R. B.; Pastore, H. O.; Environ. Sci. Technol. 2014, 48, 2472.

8. Vieira, R. B.; Moura, P. A. S.; Vilarrasa-García, E.; Azevedo, D. C. S.; Pastore, H. O.; J. CO2 Util. 2018, 23, 29.

9. Wei, J.; Shi, J.; Pan, H.; Zhao, W.; Ye, Q.; Shi, Y.; Microporous Mesoporous Mater. 2008, 116, 394.

10. Ko, Y. G.; Shin, S. S.; Choi, U. S.; J. Colloid Interface Sci. 2011, 361, 594.

11. Kishor, R.; Ghoshal, L.; A. K.; Chem. Eng. J. 2015, 262, 882.

12. Kleitz, F.; Choi, S. H.; Ryoo, R.; Chem. Commun. 2003, 2136.

13. Thommes, M.; Kaneko, K.; Neimark, A. V.; Olivier, J. P.; RodriguezReinoso, F.; Rouquerol, J.; Sing K. S. W.; Pure Appl. Chem. 2015, 87, 1051. 
14. Garcés, S. I.; Villarroel-Rocha, J.; Sapag, K.; Korili, S. A.; Gil, A.; Ind. Eng. Chem. Res. 2013, 52, 6785.

15. Qian, L.; Ren, Y.; Liu, T.; Pan, D.; Wang, H.; Chen, G.; Chem. Eng. J. 2012, 213, 186.

16. Ayada, M. M.; Salahuddina, N. A.; El-Nasra, A. A.; Torad, N. L.; Microporous Mesoporous Mater. 2016, 229, 166.

17. Sanz-Pérez E. S.; Olivares-Marín, M.; Arencibia, A.; Sanz, R.; Calleja, G.; Maroto-Valez, M. M.; Int. J. Greenhouse Gas Control. 2013, 17, 366.

18. Mello, M. R.; Phanon, D.; Silveira, G. Q.; Liewellyn, P. L.; Ronconi, C. M. Microporous Mesoporous Mater. 2011, 143, 174.

19. Quang, D. V.; Hatton, T. A.; Abu-Zahra, M. R. M.; Ind. Eng. Chem. Res. 2016, 55, 7842.

20. Lee, J.; Kim, J.-H.; Choi, K.; Kim, H.-G.; Park, J.-A.; Cho, S.-H.; Choi, J.-W.; Sci. Rep. 2018, 8.

21. Santos, A. P. B.; Santos, A. G. D.; Caldeira, V. P. S.; Guedes, A. P. M. A.; Souza, L. D.; Soares, J. M.; Araujo, A. S.; J. Therm. Anal. Calorim. 2017, 129, 391.

22. Guillet-Nicolas, R.; Ahmad, R.; Cychosz, K. A.; Kleitz, F.; Thommes, M.; New J. Chem. 2016, 40, 4351
23. Yan, X.; Zhang, L.; Zhang, Y.; Yang, G.; Yan, Z.; Ind. Eng. Chem. Res. 2011, 50, 3220 .

24. Boulaoued, A.; Fechete, I.; Donnio, B.; Bernard, M.; Turek, P.; Garin, F.; Microporous Mesoporous Mater. 2012, 155, 131.

25. Taghizadeh, M.; Akhoundzadeh, H.; Rezayan, A.; Sadeghian, M.; Int. J. Hydrogen Energy 2018, 43, 10926.

26. HeydarI-Gorji, A.; Sayari, A.; Chem. Eng. J. 2011, 173, 72.

27. Sanz, R.; Calleja, G.; Arencibia, A.; Sanz-Pérez, E. S.; Microporous Mesoporous Mater. 2012, 158, 309.

28. Aziz, B.; Hedin, N.; Bacsik, Z. Microporous Mesoporous Mater. 2012 , $159,42$.

29. Site, A. D.; J. Phys. Chem. Ref. Data 2001, 30, 187.

30. Rouquerol, F.; Rouquerol, J.; Sing, K. S. W.; Llewellyn, P.; Maurin, G.; Adsorption by powders and porous solids: Principles, methodology and applications, $2^{\text {th }}$ ed., Academic Press: San Diego, 2014.

31. Mello, M. R.; Phanon, D.; Silveira, G. Q.; Llewellyn, P. L.; Ronconi, C. M.; Microporous Mesoporous Mater. 2011, 143, 174.

32. Kim, S.-N.; Son, W.-J.; Choi, J.-S.; Ahn, W.-S.; Microporous Mesoporous Mater. 2008, 115, 497. 